\title{
PROPUESTA DE EXIGENCIAS EN LA EVALUACIÓN DE DESEMPEÑO DE MEZCLAS ASFÁLTICAS CON EQUIPO NOTTINGHAM ASPHALT MIX TESTER
}

\author{
PROPOSAL OF REQUIREMENTS IN PERFORMANCE QUALITY OF ASPHALT \\ MIXTURES WITH EQUIPMENT NOTTINGHAM ASPHALT MIX TESTER
}

\section{ROLANDO VILA ROMANÍ', GUSTAVO PATRICIO GARCÍA CAPUTI²}

\footnotetext{
Laboratorio de Carreteras de la Universidad Católica de Santiago de Guayaquil. rolando.vila@cu.ucsg.edu.ec

2 Laboratorio de Carreteras de la Universidad Católica de Santiago de Guayaquil. gustavo.garcia@cu.ucsg.edu.ec
}

RESUMEN

Internacionalmente existe una marcada tendencia al empleo de diferentes pruebas de desempeño para mejorar los controles de calidad a las mezclas asfálticas. Si bien, en el Ecuador, desde el año 2000, las especificaciones constructivas establecen el empleo de algunas para tráfico pesado, en la práctica, esta consideración no fue respetada debido al escaso y costoso equipamiento necesario en el país y a la falta de unas exigencias claras de calidad con dichas pruebas. En este trabajo se proponen exigencias que deben cumplir las mezclas asfálticas cuando se le realizan pruebas de desempeño con el equipo Nottingham Asphalt Mix Tester (NAT). Esta propuesta se plantea y fundamenta a través de una investigación desarrollada en el Laboratorio de Carreteras (Suelos y Asfaltos) de la Universidad Católica de Santiago de Guayaquil, que comprendió el estudio experimental de mezclas producidas en 15 plantas y 9 pavimentos flexibles, correspondientes a las diferentes regiones del Ecuador. La propuesta incluye rangos para la evaluación de los módulos de rigidez y de las deformaciones plásticas; así como un procedimiento para el análisis y calificación de la fatiga en el control de calidad de las mezclas asfálticas.

PALABRAS CLAVE: mezclas asfálticas, módulo de rigidez, deformación plástica, fatiga.
Worldwide there is a tendency to use different performance tests to improve quality control in asphalt mixtures. In Ecuador, since 2000, the construction specifications establish the use of these performance tests for heavy traffic. However, this consideration has not been respected due to the limited and expensive equipment needed in the country and the lack of clear quality requirements to meet with those tests. In this paper, a list of requirements to be met by asphalt mixtures during performance tests using the Nottingham Asphalt Mix Tester (NAT) equipment is proposed. This proposal is based on research conducted at the Laboratorio de Carreteras (Suelos y Asfaltos), of the Universidad Católica de Santiago de Guayaquil, which included the experimental study of mixtures produced in 15 plants and 9 flexible pavements, corresponding to the diferent regions of Ecuador. The proposal includes the evaluation of stiffness modulus and plastic deformations within a range, as well as a method of analysis and rating of fatigue on quality control of asphalt mixtures.

KEYWORDS: asphalt mixtures, stiffness modulus, permanent deformation, fatigue. 
INTRODUCCIÓN

Desde hace dos décadas, aproximadamente, en los países desarrollados se emplean pruebas adicionales de desempeño para el control de calidad de las mezclas asfálticas, lo que permite un buen control de lo que será su comportamiento en obra, ante las fallas por fatiga y ahuellamiento, entre otras.

A mediados de los años noventa en los EE.UU. comenzó a emplearse el denominado sistema Superpave (Asphalt Institute, 1996). Este sistema está constituido por tres componentes básicos: (1) especificaciones para los ligantes asfálticos basadas en su desempeño; (2) un procedimiento de diseño de mezclas y (3), técnicas de análisis de mezclas para predecir su desempeño. Estas últimas técnicas para mezclas basadas en dos dispositivos: el equipo de corte de Superpave (SST) y el equipo de tracción indirecta (IDT), dada su complejidad y la falta de consenso acerca de sus capacidades para predecir el desempeño, sólo se han implementado de manera limitada para algunos proyectos experimentales $u$ otras aplicaciones especiales (Mc Daniel, 2007). En los EE.UU. se han desarrollado como alternativa ante esta situación las llamadas pruebas simples de desempeño (SPT) (Witczak, 2002). En la actualidad, las SPT comprenden básicamente: módulo dinámico a compresión, tiempo de flujo y número de flujo (Vázquez, 2009).

En la Unión Europea, los requerimientos generales de las mezclas asfálticas se recogen en la normativa CEN EN 13108-1 (2008), que maneja dos enfoques: el empírico, basado en controles volumétricos de susceptibilidad al agua y ensayo en pista; y el fundamental, mediante pruebas de desempeño, donde se controla la rigidez, la resistencia a la deformación permanente en prueba a compresión cíclica triaxial y la resistencia a la fatiga sobre vigas trapezoidales o prismáticas.

Un primer intento en llamar la atención sobre estos aspectos en el Ecuador fue la inclusión en las especificaciones técnicas publicadas en el año 2000 (MTOP, 2000) del criterio de que, para tráfico muy pesado, las mezclas para capa de rodadura deben ser sometidas a un estudio detallado que incluya sus módulos de rigidez y la evaluación del comportamiento ante las deformaciones y la fatiga. Posteriormente, en las especificaciones técnicas del 2002 que se encuentran vigentes, se mantuvo este criterio (MTOP; 2002). En la práctica, esta consideración no fue respetada debido al escaso y costoso equipamiento necesario en el país y la falta de exigencias claras de calidad a cumplir con dichas pruebas.

El objetivo de este estudio es proponer exigencias que deberían cumplir las mezclas asfálticas cuando se le realizan pruebas de desempeño con el equipo Nottingham Asphalt Mix Tester (NAT). Esta propuesta se plantea y fundamenta a través de una investigación desarrollada en el Laboratorio de Carreteras (Suelos y Asfaltos) de la Universidad Católica de Santiago de Guayaquil.

\section{ESTUDIO DE MEZCLAS ASFÁLTICAS CON PRUEBAS ADICIONALES DE DESEMPEÑO}

El equipo NAT modelo NU-10 existente en nuestro laboratorio permite realizar varias pruebas de desempeño. El NAT aplica las cargas mediante un sistema neumático con servo-control, midiéndose la fuerza aplicada por el transductor de la celda de carga. El equipo utiliza una computadora junto con un sistema digital de adquisición de datos y control de sistema que permite desarrollar las distintas pruebas y procesar la información a través de programas específicos. Con el NAT se pueden realizar diferentes tipos de pruebas: módulos de rigidez bajo deformación o carga controlada, compresión cíclica uniaxial sin confinamiento (creep dinámico) y pruebas de fatiga bajo dispositivo de tracción indirecta. (Ver Figura 1).

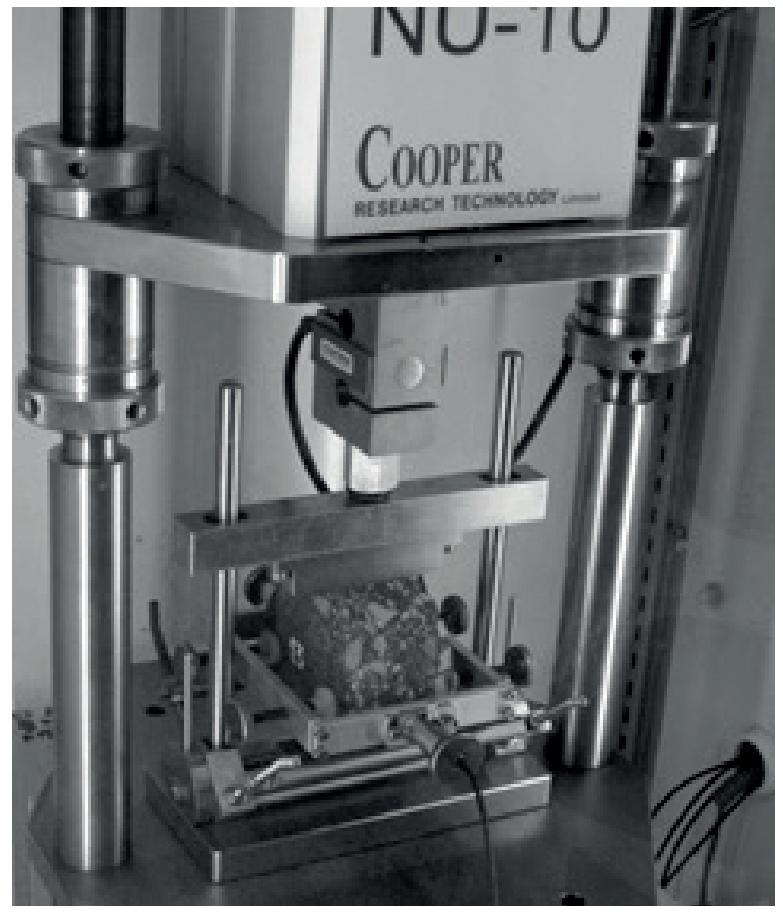

Figura 1. Equipo NAT en una prueba de módulo de rigidez. 
Para la realización de estas pruebas en general, se consideran las metodologías dadas por el fabricante del equipo (Cooper, 2002), que coinciden con los del Comité Europeo de Normalización (CEN, 2001) respecto a la configuración de cargas y al sistema de medición y control; aunque para el caso de la prueba de compresión cíclica uniaxial la CEN establece cierto confinamiento de la muestra, ya que el plato de carga tiene un diámetro menor que el de la muestra cilíndrica a ensayar.

A continuación se muestran los resultados obtenidos en el proyecto de investigación: "Propuesta de exigencias de calidad y espesores de mezclas asfálticas para los pavimentos de las diferentes regiones del Ecuador" (Vila et al, 2015). Vale destacar que, de acuerdo con el objetivo de esta publicación, solo se exponen los aspectos relacionados con las exigencias de calidad.

Las propuestas que se realizan se obtienen del procesamiento de la información correspondiente a pruebas de desempeño realizadas sobre:

1. Núcleos de carpeta asfáltica extraídos en 9 vías de las distintas regiones del país: Costa, Sierra y Amazonía, con tiempo de colocación entre tres y cuatro años y con diferentes niveles de deterioros: bajo, regular y alto para cada región. Si bien dichas mezclas se construyeron con diferentes agregados y similar tipo de asfalto (AC-20 de la Refinería de Esmeraldas), en todos los casos se debieron cumplir las exigencias convencionales de calidad para los diferentes materiales. Se extrajeron 12 núcleos de cada vía, tomados en una misma banda de pavimentación, lo que normalmente coincide con el ancho de un carril, espaciado cada cinco metros aproximadamente.

2. Briquetas confeccionadas con 15 tipos de mezclas asfálticas densas fabricadas en diferentes plantas de las tres regiones, empleando distintos agregados minerales. Para cada una de las mezclas se varió la energía de compactación a nivel de laboratorio, aplicando diferentes números de golpes por capa (75, 50 y 35 golpes). En todos los casos se utilizó el mismo tipo de cemento asfáltico, clasificado como AC-20, procedente de la Refinería de Esmeraldas.
MÓDULOS DE RIGIDEZ ANÁLISIS

El propósito de obtener un valor de rigidez es proveer información sobre las propiedades de un material, de manera que las respuestas tenso-deformacionales formando parte de un pavimento, bajo las cargas aplicadas, puedan ser determinadas. Debido a que la rigidez no es una medida de la resistencia, una mezcla asfáltica con un alto valor de rigidez no necesariamente tendrá una alta resistencia (NCAT, 2009). Un alto valor de rigidez indica que bajo un esfuerzo aplicado dado existirá una baja deformación en la mezcla, pero eso aún no significa una alta resistencia. Por ejemplo, un material frágil puede tener un alto valor de rigidez pero baja resistencia y un material muy flexible puede tener una baja rigidez pero mayor resistencia.

Mezclas muy rígidas en la capa asfáltica reducirán los esfuerzos en las capas inferiores que conforman el pavimento y el nivel de deformación en la parte inferior de la capa asfáltica, pero también incrementarán la sensibilidad de la mezcla a fatigarse.

Por otra parte, el ahuellamiento o deformación permanente en las mezclas asfálticas es afectada por su rigidez, sin embargo, no puede ser estimada solamente a partir de la misma, habiéndose demostrado la necesidad de pruebas especiales como las ruedas cargadas, etc. En general, las pruebas para determinar la rigidez, como el módulo resiliente o el módulo dinámico, emplean niveles bajos de carga o deformación que no movilizan la estructura del agregado en la mezcla, lo cual es necesario para un análisis serio del ahuellamiento.

$\mathrm{Su}$ evaluación en el equipo NAT se realizó a la temperatura de $20^{\circ} \mathrm{C}$, empleando un nivel de deformación controlada de cinco micrones y ondas sinusoidales con intervalo de tiempo entre el inicio del pulso de carga y el punto en el que la carga es máxima, de 0,12 segundos.

\section{CONCLUSIONES SOBRE LOS MÓDULOS DE RIGIDEZ}

1. Existe la necesidad de realizar en vías de tráfico pesado un control de la rigidez de la mezcla como punto inicial para conocer su respuesta ante los esfuerzos y deformaciones, lo que permitirá además su inserción seria en el diseño de pavimentos.

2. Se recomienda como un primer paso hacia el establecimiento de exigencias sobre mezclas recién producidas en planta, considerando los módulos de rigidez bajo 
deformación controlada a $20^{\circ} \mathrm{C}$ evaluados con el equipo NAT el siguiente criterio:

Mezclas satisfactorias: 3.000 a 4.000

$\mathrm{MPa}$

Mezclas tolerables: 2.500 a $3.000 \mathrm{MPa}$ y 4.000 a $5.000 \mathrm{MPa}$

Mezclas inadecuadas: menores a 2.500 MPa ó mayores a $5.000 \mathrm{MPa}$

En la aplicación de este criterio, se puede introducir un envejecimiento en horno, de plazo corto, como el que se utiliza en el diseño de mezclas Superpave, en el que se envejece la mezcla de laboratorio en horno a $135^{\circ} \mathrm{C}$ por cuatro horas, antes de preparar los especímenes. Para mezclas preparadas en planta, este tiempo se reduce a dos horas o una hora, de manera que refleje lo que ocurre hasta que se coloca la mezcla en obra. El límite alto corresponde a mezclas densas calientes, preparadas con asfalto AC-20 de la Refinería Esmeraldas. Si se tratara de mezclas especialmente diseñadas para producir módulos de rigidez altos que se aprovechen en el espesor de diseño, deberá comprobarse una apropiada resistencia a la fatiga.

\section{FATIGA \\ ANÁLISIS}

Los agrietamientos por fatiga en la capa asfáltica son considerados un problema estructural donde además de la calidad de la mezcla incide grandemente la rigidez de la capa que soporta a la misma. Por esta razón en los estudios realizados en las vías además de inspecciones de daños se hizo una evaluación estructural deflectométrica con un equipo Falling Weight Deflectometer (FWD). Ver Figura 2. El procesamiento de las deflexiones se realizó aplicando el Método Yonapave (Hoffman, 2003).

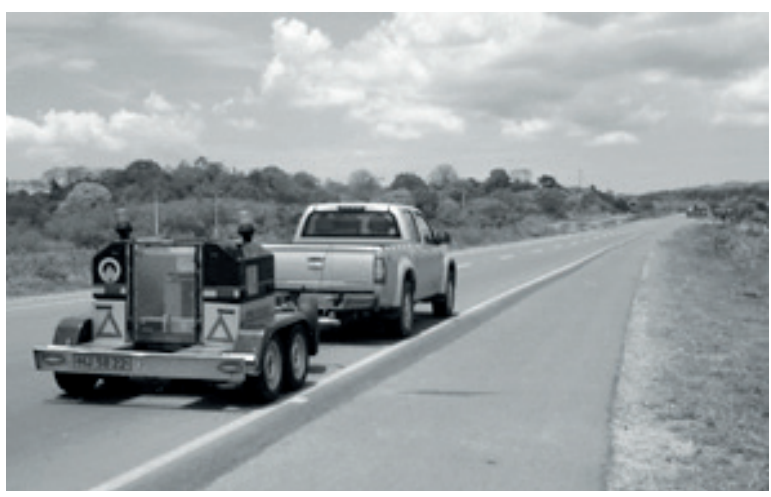

Figura 2. Medición con equipo FWD en la vía "Progreso-Playas".
La prueba de fatiga se realizó por el método diametral o de tracción indirecta, aplicado a la briqueta un esfuerzo controlado. Previamente, tiene que determinarse el módulo de rigidez bajo el mismo esfuerzo. Con la prueba de fatiga se obtiene el número de aplicaciones de carga requeridas para alcanzar la falla o una deformación máxima de $5 \mathrm{~mm}$. El tiempo de carga es de 120 milisegundos y la temperatura para el estudio fue de $20^{\circ} \mathrm{C}$. Los resultados suelen representarse en gráficas log-log, relacionando el número de ciclos para el fallo (Nf) en el eje de las abscisas con las deformaciones unitarias $(\varepsilon)$ en el eje de las ordenadas.

\section{COMPARACIÓN ENTRE LA FATIGA DE NÚCLEOS DE VÍAS Y BRIQUETAS DE PLANTAS}

Los resultados se resumen a continuación en la Tabla 9 y en la Figura 8. Considerando las ecuaciones típicas de fatiga para una misma temperatura con la forma:

$$
\varepsilon=\mathrm{a} \mathrm{Nf}-\mathrm{b}
$$

siendo:

$\varepsilon$ : deformación unitaria (microstrain)

$\mathrm{Nf}$ : repeticiones para el fallo

Se incluyen en dicha tabla los coeficientes "a" y "b".

TABLA 1. DEFORMACIONES CORRESPONDIENTES A LOS PROMEDIOS Y PERCENTILES 15

\begin{tabular}{|c|c|c|c|c|}
\hline \multirow{3}{*}{ REPETICIONES } & \multicolumn{2}{|c|}{ VÍAs } & \multicolumn{2}{|c|}{ PLANTAS } \\
\hline & PERCENTIL & PROMEDIO & PERCENTIL & PROMEDIO \\
\hline & \multicolumn{4}{|c|}{ DEFORMACIONES UNITARIAS (MICROSTRAIN) } \\
\hline 500 & 230 & 353 & 386 & 507 \\
\hline 1000 & 207 & 298 & 337 & 403 \\
\hline 5000 & 154 & 202 & 184 & 239 \\
\hline 10000 & 135 & 171 & 147 & 191 \\
\hline 25000 & 110 & 138 & 105 & 143 \\
\hline 50000 & 89 & 118 & 79 & 115 \\
\hline 100000 & 70 & 101 & 59 & 93 \\
\hline \multicolumn{5}{|c|}{ COEFICIENTES DE LAS LEYES DE FATIGA } \\
\hline a & 947,53 & 1523,73 & 3850,08 & 3687,49 \\
\hline b & 0,218 & 0,237 & 0,359 & 0,320 \\
\hline
\end{tabular}




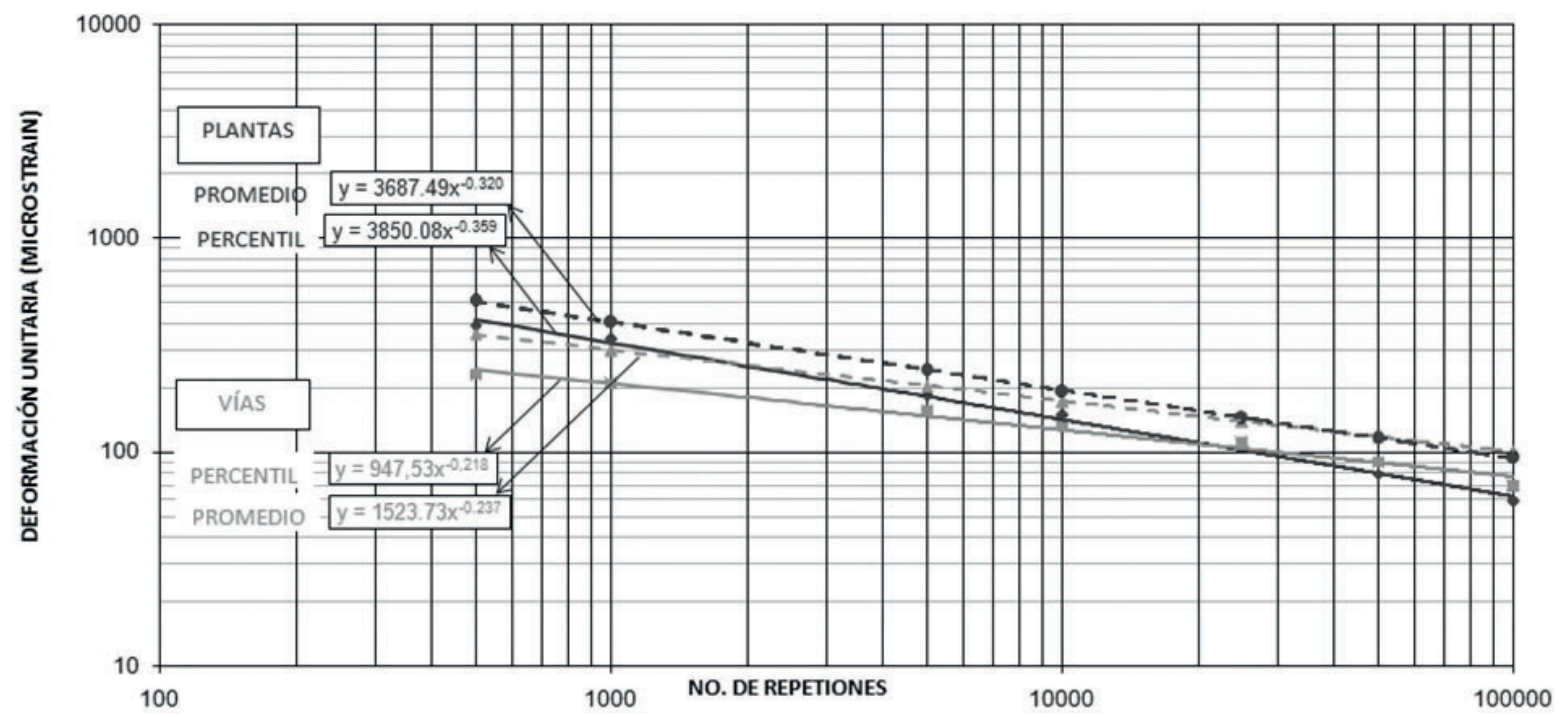

Figura 3. Relaciones Nf vs $\varepsilon$ correspondientes a promedios y percentiles 15 .

\section{PROPUESTA DE PROCEDIMIENTO DE PRUEBA RÁPIDA} PARA LA EVALUACIÓN DE LA TENDENCIA A LA FATIGA

Esta prueba se está aplicando de manera introductoria en controles a briquetas y núcleos en nuestro laboratorio. Consta de los siguientes pasos:

1. Obtención inicial del módulo rigidez bajo deformación controlada (Sm).

2. Cálculo del esfuerzo ( $\sigma$ en $\mathrm{kPa}$ ) considerando un valor de deformación unitaria (ع) entre 100 y 150 microstrain y un coeficiente de Poisson $(\mu)$ igual a 0.35 .

$$
\sigma(K P a)=\frac{S m(K P a) \varepsilon}{(1+3 \mu)}
$$

3. Aproximar el esfuerzo calculado a los permitidos por el NAT para los ensayos de módulos bajo carga controlada. Por ejemplo esfuerzos de 300, 350 ó $400 \mathrm{KPa}$.

4. Con dicho esfuerzo aproximado realizar una prueba de módulo de rigidez bajo carga controlada para precisar la deformación unitaria inicial.

5. Realizar el ensayo de fatiga con el esfuerzo del Paso 4 para determinar el número de repeticiones para el fallo.

6. El comportamiento estimado de la mezcla estará dado por la ubicación del punto $(\mathrm{Nf}, \varepsilon \mathrm{o})$ respecto a las líneas de control de planta. Ver Tabla 1 y Figura 3.

\section{CONCLUSIONES SOBRE LA FATIGA}

1. Existe la necesidad de realizar, para vías de tráfico pesado, un control de la mezcla para evaluar su posible desempeño a la fatiga, si bien este comportamiento también está relacionado con la rigidez de la capa que soporta dicha mezcla. Se detectaron "buenas" mezclas fatigadas debido a un bajo espesor de capa y/o a números estructurales bajos.

2. Se recomienda como un primer paso hacia el establecimiento de exigencias respecto a la fatiga mediante pruebas con el NAT, especialmente para los controles de calidad sobre mezclas recién producidas en planta:

a. Ejecutar pruebas de fatiga sobre 1 ó 2 briquetas en valores de esfuerzo que correspondan con una deformación unitaria inicial de 100 a 150 microstrain y determinar su ubicación en un gráfico Nf vs $\varepsilon$.

b. Trazar en dicho gráfico las líneas de tendencia correspondientes al percentil $15 \mathrm{y}$ al promedio obtenidas, en una primera aproximación, para las mezclas de plantas. Ver Tabla 1 y Figura 3.

c. Si los puntos para la mezcla en particular analizada caen por debajo de la línea del percentil, el comportamiento estimado será inadecuado. Si los puntos se ubican por arriba de la línea del promedio, el comportamiento estimado será satisfactorio. Entre ambas líneas el comportamiento no puede garanti- 
zarse como satisfactorio, aunque puede considerarse como tolerable mientras se desarrollan más estudios que aporten a la reducción de este umbral.

3. El funcionamiento del criterio anterior será válido siempre que la capa asfáltica a construir responda a un diseño de pavimento, ya sea nuevo o para rehabilitación, correctamente ejecutado y a partir de estudios serios de los materiales involucrados. Se puede definir la deflexión característica requerida en la capa por debajo de la carpeta y, según el espesor y módulo de la carpeta, determinar si va a haber la resistencia necesaria a la fatiga.

4. La propuesta de prueba rápida de fatiga es interesante y rigurosa en su funcionamiento, y puede utilizarse de manera referencial para corregir diseños de mezclas y optimizar esta propiedad. Al mismo tiempo, se va a ganar más experiencia en su utilización, lo que permitirá la validación de sus resultados, así como el ajuste de los criterios de desempeño (ubicación de la línea de aceptación de resultados).

\section{DEFORMACIÓN PERMANENTE O PLÁSTICA} ANÁLISIS

Se empleó la prueba de compresión cíclica uniaxial para estimar el comportamiento ante las deformaciones plásticas. En esta prueba un ciclo de carga consiste en la aplicación de una tensión durante 1 segundo seguido por 1 segundo de descanso, con ondas cuadráticas. El ensayo se realizó a una temperatura de $40^{\circ} \mathrm{C}$, con una magnitud de carga de $100 \mathrm{KPa}$, midiendo las deformaciones verticales provocadas por 3600 repeticiones de dicha carga.

VALIDACIÓN

El Laboratorio de Carreteras de la UCSG realizó hace dos años el estudio de un tramo de la carretera principal E-25, Troncal de la Costa, donde existían sectores que presentaban diferentes niveles de ahuellamiento, particularmente en una capa de refuerzo (recapeo) con espesor de $7.5 \mathrm{~cm}$.

Se contrastaron los niveles máximos de los ahuellamientos medidos en 31 puntos con los resultados de las pruebas de compresión cíclica uniaxial, en núcleos extraídos en los mismos sitios de la medición. Ver Figura 4.

En general, el criterio analizado de que mezclas con niveles de deformación menores o iguales al $1 \%$ presentan ahuellamientos inferio- res a $12 \mathrm{~mm}$, máximo admitido por el MTOP, se cumple en el $96.8 \%$ de los casos, por lo que se considera válido para emplearse en el Ecuador. Este estudio fue publicado en la UCSG. (Vila, 2013).

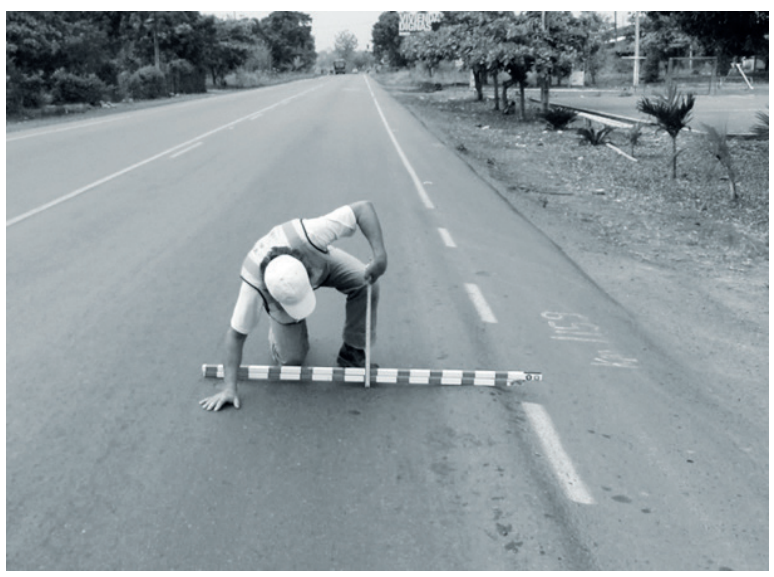

Figura 4. Medición del ahuellamiento en la vía E - 25.

\section{CONCLUSIONES SOBRE LAS DEFORMACIONES PERMANENTES}

1. Existe la necesidad de realizar en vías de tráfico pesado un control del problema potencial de la deformación permanente de las mezclas. Por ejemplo, en siete de las 15 plantas analizadas la deformación superó el $1 \%$, aunque pudiera argumentarse que algunas de esas mezclas no corresponderían a vías de tráfico pesado.

2. Se recomienda como un primer paso hacia el establecimiento de exigencias sobre mezclas recién producidas en planta, considerando el porcentaje de deformación determinado mediante la prueba de compresión cíclica uniaxial a $40^{\circ} \mathrm{C}$ sobre briquetas tipo Marshall, el siguiente criterio:

- Mezclas satisfactorias: valores menores o iguales a $1 \%$.

- Mezclas inadecuadas: valores mayores a $1 \%$.

\section{DISCUSIÓN}

Todos los criterios de control de calidad analizados mediante pruebas de desempeño, es decir: módulo de rigidez, fatiga y deformación permanente por compresión cíclica uniaxial (creep dinámico); corresponden a pruebas que pueden realizarse con una misma máquina, el NAT, cambiando solo los cabezales y empleando los programas correspondientes.

El uso de otras tecnologías para evaluar los desempeños es posible, incluso convendría en 
algunos casos; pero necesitaría de igual forma estudios que permitan un mínimo de seguridad en su manejo en nuestras condiciones.

Por la cantidad de información manejada en esta investigación, más las experiencias acumuladas en el Ecuador con el equipo NAT, no se estima oportuno, de manera inicial, establecer exigencias particulares para cada una de las diferentes regiones geográficas.

Se consideran factibles de emplear las exigencias propuestas con el equipo NAT, para mezclas recién producidas en planta, pero con un envejecimiento en horno de plazo corto, como el utilizado en el diseño de mezclas Superpave. Estas exigencias se han precisado en las conclusiones correspondientes a cada prueba.

\section{RECOMENDACIONES}

1. Emplear la prueba rápida de fatiga propuesta para corregir diseños de mezclas y controles de calidad.

2. Limitar para las vías de tráfico pesado el empleo de arenas naturales en las mezclas. Debe cuidarse además el cumplimiento de la exigencia de caras fracturadas en los agregados, en especial aquellos provenientes de la trituración de cantos rodados de río.

3. Prestar atención a los bajos contenidos de fillers en nuestras mezclas y a su calidad, así como a las relaciones filler/betún que deben cumplirse. En este caso el contenido de betún es el efectivo.

4. Evitar el empleo de asfaltos sobrecalentados en planta por su incidencia en la fatiga.

5. Aplicar sistemáticamente, en los controles de calidad, la evaluación del daño en agua mediante la prueba de tracción indirecta con carga monotónica.

\section{REFERENCIAS BIBLIOGRÁFICAS}

Asphalt Institute. (1996): Antecedentes del Diseño y Análisis de Mezclas Asfálticas de SUPERPAVE. Traducción del I.P.C. Lexington, KY.

Cooper Research Technology Limited (2002): NU10 \& NU- 5. Operation \& Maintenance Manual. United Kingdom.
European Committee of Standardization (2001): CEN 12697. Bituminous mixtures. Test methods for hot mix asphalts. Part 25-26. English version. Brussels.

European Committee of Standardization (2008): CEN-EN 13108-1. Bituminous Mixtures - Material Specifications - Part 1: Asphalt Concrete - Incorporating Corrigendum January 2008. English version. Brussels.

Hoffman, M.S. (2003): Direct Method for Evaluating Structural Needs of Flexible Pavements with Falling-Weight Deflectometer. Transportation Research Record. Volume 1860. T.R.B. pp. 41-47.

McDaniel, R.S. (2007): Current Superpave Mix Design Practice. Transportation Research Circular Number E-C 124. T.R.B. pp. 2-11.

Ministerio de Obras Públicas y Comunicaciones (2000): Especificaciones Técnicas para la Construcción de Carreteras y Puentes. Quito.

Ministerio de Obras Públicas y Comunicaciones (2002): Especificaciones Técnicas para la Construcción de Carreteras y Puentes. Quito.

NCAT (National Center for Asphalt Technology). (2009). Hot Mix Asphalt Materials, Mixture Design and Construction. Third Edition. $\mathrm{Au}^{-}$ burn University.

Vazquez, C.C. et al. (2009): Laboratory Evaluation of Influence of Operational Tolerance (Acceptance Criterion) on Performance of HotMix Asphalt Concrete. CTR Technical Report: 0-6045-1. Center for Transportation Research at The University of Texas at Austin.

Vila, R. (2013): Deformaciones plásticas en la capa asfáltica de una vía primaria en la región costa del Ecuador. Revista Alternativas. Universidad Católica de Santiago de Guayaquil. (No. 18): 3-9.

Vila, R.; García G. (2015): Informe Final. Proyecto de Investigación: "Propuesta de exigencias de calidad y espesores de mezclas asfálticas para los pavimentos de las diferentes regiones del Ecuador". SINDE. Universidad Católica de Santiago de Guayaquil.

Witczak, M.W. et al. (2002): Simple Performance Test for Superpave Mix Design. NCHRP Report 465. Transportation Research Board. Washington, D.C. 